\title{
A Numerical Study of Time-Domain Model Validation for Robust Control
}

\author{
Sunil L. Kukreja ${ }^{\dagger}$, Johan Löfberg ${ }^{\dagger}$ and Benoit Boulet ${ }^{\ddagger}$ \\ ${ }^{\dagger}$ Div. of Automatic Control, Dept. of Elect. Eng., Linköpings universitet \\ ${ }^{\ddagger}$ Dept. of Elect. Eng., CIM, McGill University, E-mail: boulet@cim.mcgill.ca
}

\begin{abstract}
In this paper, we investigate a discrete-time approach to uncertainty modeling for robust control. We show via simulations of one LTI system and experimental data from a onetank test-bed that a current technique for time-domain uncertainty modeling leads to a feasible linear program. Hence, it is useful for developing robust control solutions.
\end{abstract}

\section{Introduction}

Model validation is an important step in developing strategies for robust control. This step is typically preceded by system identification, as well as, system analysis and physical modeling. Model validation is concerned with assessing whether a given nominal model can reproduce data from a plant, collected after some initial experiments to obtain estimation data [1]. The model validation problem is really one of model invalidation since a given model can only be said to be not invalidated with the current evidence. Future evidence may invalidate the model.

The motivation for this study is to investigate whether a time-domain approach to uncertainty modeling for linear time-invariant (LTI) and linear time-varying (LTV) systems developed by Poola et al. [4] can be implemented for robust control applications. While the work provided theoretical development for their approach to uncertainty modeling, it did not provide any simulated example or experimental application to verify their theory is numerically tractable.

To date, the authors are not aware of any simulation study or application to experimental data of Poola et al.'s [4] work. However, a sampled-data approach to model validation developed in [2] was successfully tested by simulation [2] and experimental data [5]. As such, in this paper, we investigate the feasibility of the approach of [4] to experimental data by first studying the behavior of this technique on one simulated causal, LTI system for $\ell_{1}$ control and applying this approach to experimental data from a one-tank test-bed.

Our results show, this approach to uncertainty modeling provides a numerically tractable solution for noisy simulated data as well as for experimental data.
2 Problem Statement

We considered the class of uncertainty models described by an output multiplicative uncertainty, as

$y(k)=\left(G_{0}+G_{0} W \Delta\right) u(k)+d(k) ; d$ is in a convex set

where $u(k)$ and $y(k)$ are the input-output measurements, $G_{0}$ a causal, LTI nominal model, $W$ a causal, LTI normalized uncertainty filter, $\Delta \in \ell_{1}$ a normalized system uncertainty and $d \in D:=\left\{d \in \ell_{\infty}[0, \ldots, M-1]:\|d\|_{\infty} \leq 1\right\}$, a noise or disturbance acting on the system. The uncertainty filter $W$ is selected so that $|W(j \omega)|$ is an upper bound on $\left|\frac{\left.\left.G_{p} j \omega\right)-G_{0} j \omega\right)}{\left.G_{0} j \omega\right)}\right|$ where $G_{p}$ is the perturbed model. Although $\Delta$ is assumed norm-bounded in $\ell_{1}$, this uncertainty structure was selected because it may be capable of describing model mis-specification due to unmodeled dynamics, noise and other disturbances. [6].

Therefore, the model validation problem for this class of uncertainty is stated as [4]: Given input-output sequences $u=\left\{u_{0}, u_{1}, \ldots, u_{N-1} \in \mathfrak{R}\right\}$ and $y=\left\{y_{0}, y_{1}, \ldots, y_{N-1} \in \mathfrak{R}\right\}$ there exists a stable causal, LTI operator $\Delta$ with

$$
\|\Delta\|_{i \infty} \leq \gamma \quad \text { where }\|\cdot\|_{i \infty} \text { denotes } \ell_{\infty} \text { - induced }
$$

such that $\Delta\left(\hat{u}_{0}, \hat{u}_{1}, \ldots, \hat{u}_{N-1}, *, *, \ldots\right)=\left(\hat{y}_{0}, \hat{y}_{1}, \ldots, \hat{y}_{N-1}\right.$, $*, *, \ldots)$ if and only if the following linear programming problem is feasible [4]

$$
\mathbf{L P}(\hat{u}, \hat{y}-q, \gamma),
$$

where $\hat{u}(k)=\pi_{N} W G_{0} u(k), \hat{y}(k)=y(k)-\pi_{N} G_{0} u(k)$ and $q(k)$ the system errors which encompass both the effect of model uncertainty and noise in $\pi_{N} D$ and $\pi_{N}$ is the truncation operator keeping $N$ data points [4]. Since the uncertainty filter is normalized the criterion for assessing model invalidation is $\gamma<1$.

\section{Procedures}

The efficacy of this model invalidation algorithm was assessed using (i) noise corrupted data from a simulated second-order system but nominal model identified as a firstorder model and (ii) experimental data from a one-tank testbed. The simulated system, $H_{1}(z)$, and nominal model, $\hat{H}_{1}(z)$, used in study are

$$
H_{1}(z)=\frac{0.2 z^{-1}+0.08 z^{-2}}{1-0.42 z^{-1}-0.32 z^{-2}} \quad \hat{H}_{1}(z)=\frac{b_{1} z^{-1}}{1-a_{1} z^{-1}} .
$$


First, we assessed the performance of this technique using twenty Monte-Carlo simulations in which each inputoutput realization was unique, and had a unique, white, zeromean, random noise sequence added to the output; with $\ell_{\infty}-$ norm $\leq 0.1(\mathrm{SNR} \approx 16 \mathrm{~dB})$. Each input was uniformly distributed, white, zero-mean, random sequences with unit variance.

Second, a single tank test-bed was used to assess the feasibility of this approach for control applications. The test-bed is a single tank system, described in detail in various literature (see e.g., [3]). We modeled this one-tank process as a firstorder LTI system. The form of the identified model was the same as given on the right side of Eqn. 4. Two data sets were collected for this study. The measured data was anti-alias filtered with an fourth-order $10 \mathrm{~Hz}$ Bessel filter and sampled at $100 \mathrm{~Hz}$ by a 16-bit A/D converter. After recording, the experimental data was decimated by a factor of 3 , resulting in a final sampling rate of $33 \mathrm{~Hz}$.

For both the simulated and experimental case, an initial data set was generated to estimate a nominal model. The System Identification toolbox in Matlab was used to identify a nominal model. Next, the system was perturbed with a fresh input set to generate data for validation. The LMI toolbox in Matlab was used to solve the linear programming problem. The uncertainty filter, $W$, was computed by assuming a 33\% uncertainty for each parameter. Specifically, we randomly selected 100 parameter sets for the perturbed model, $G_{p}$, computed the Bode plot for each then selected the system which gave the best coverage of the uncertainty region as our uncertainty filter. The selected uncertainty filter was multiplied by a small gain factor so the filter could contain the entire uncertainty region. For identification $N_{e}=5,000$ points were used and to determine feasibility of the model invalidation problem $N_{v}=200$ were used.

\section{Results}

\subsection{Simulated Data}

We first studied the simulated case since if this approach fails for a simulated system it is likely to fail with experimental data. Fig. 1 (left panel) shows the results for this simulated study. The plot shows that $\gamma$ was close to zero, for all realizations. We expected $\gamma$ to be less than 1 in this case since we selected our uncertainty filter, $W$, to fully cover the uncertainty region.

\subsection{Experimental Data}

Next, we studied how this technique performs with experimental data to access the feasibility of using this approach in an industrial setting. Figure 1 (right panel) shows the results of this study. The figure shows, with experimental data, this model invalidation procedure provided a $\gamma<1$.

\section{Conclusion}

Our results demonstrate that for a simulated system with unmodeled dynamics this time-domain approach to model in-
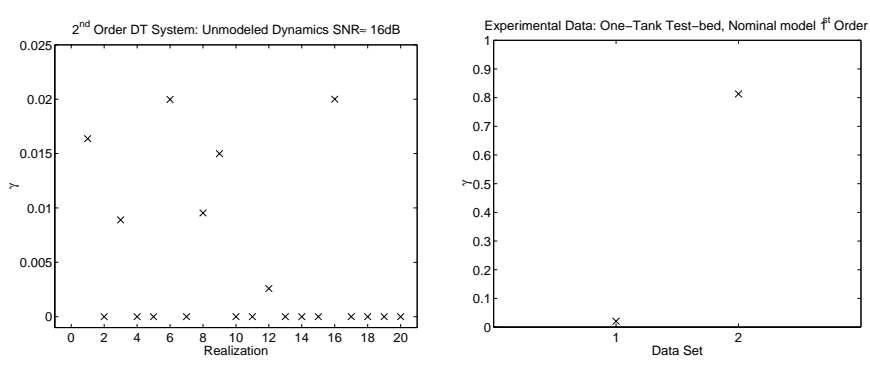

Figure 1: Feasibility of model invalidation. Nominal model: first-order. (Left) True system: simulated second-order system $\left(N_{v}=200\right)$. (Right) Experimental data set $\left(N_{v}=500\right)$.

validation does provide a good and stable estimate of the system uncertainty. The small $\gamma$ 's in Fig. 1 are due to our uncertainty filter's ability to explain the unmodeled dynamics. It would be worth considering the case where there are unmodeled dynamics and an uncertainty filter that does not cover the uncertainty well to assess the performance of this algorithm.

For experimental data this approach provided a reliable $\gamma$. Although our results indicate that this approach for model validation is practical for designing control strategies for real systems, we believe that the results of Poola et al. [4] have not been thoroughly tested with experimental data. We have not assessed the performance of this time-domain approach for $H_{\infty}$ control. This is being examined for timedomain data and the results will be give at a future date.

\section{Acknowledgments}

Supported by grants from NSERC Canada.

\section{References}

[1] B. Boulet and B.A. Francis. Consistency of openloop experimental frequency-response data with coprime factor plant models. IEEE Trans. Automatic Control, 43(12):1680-1691, 1998.

[2] L. Chen and R. Smith. Closed-loop model validation; an application to an unstable experimental system. In Proc. ACC, volume 17, pages 618-622, Philadelphia, U.S.A., June 1998.

[3] B.A. Ogunnaike and W.H. Ray. Process Dynamics, Modeling, and Control. Oxford University Press, New York, first edition, 1994.

[4] K. Poolla, P.P. Khargonekar, A. Tikku, J. Krause, and K. Nagpal. A time-domain approach to model validation. IEEE Trans. Automatic Control, 39(5):951-959, 1994.

[5] R. Smith and D.E. Dullerud. Continuous-time control model validation using finite experimental data. IEEE Trans. Automatic Control, 41(8):1094-1105, 1996.

[6] K. Zhou. Essentials of Robust Control. Prentice Hall PTR, Englewood Cliffs, New Jersey, first edition, 1997. 